\title{
GEMINGA'S SOFT X-RAY EMISSION AND THE STRUCTURE OF ITS SURFACE
}

\author{
Dany Page \\ Instituto de Astronomía, UNAM, Apdo Postal 70-264, 04510 México D.F., México. \\ page@astroscu.unam.mx \\ Yu. A. Shibanov \\ Ioffe Physical-Technical Institute, St Petersburg 194021, Russia. \\ shib@ammp.ioffe.rssi.su \\ and \\ V. E. Zavlin \\ Max Planck Institut für Extraterrestrische Physik, Garching, Germany. \\ zavlin@rosat.mpe-garching.mpg.de
}

\begin{abstract}
We present a model to explain the decrease in the amplitude of the pulse profile with increasing energy observed in Geminga's soft X-ray surface thermal emission. We assume the presence of plates surrounded by a surface with very distinct physical properties: these two regions emit spectra of very distinct shapes which present a crossover, the warm plates emitting a softer spectrum than the colder surrounding surface. The strongly pulsed emission from the plates dominates at low energy while the surroundings emission dominates at high energy, producing naturally a strong decrease in the pulsed fraction. In our illustrative example the plates are assumed to be magnetized while the rest of the surface is field free.

This plate structure may be seen as a schematic representation of a continuous but very nonuniform distribution of the surface magnetic field or as a quasi realistic structure induced by past tectonic activity on Geminga.
\end{abstract}

Subject headings: pulsars: individual (Geminga) — stars: neutron stars: X-rays — pulsars: general

Submitted to The Astrophysical Journal Letter. 


\section{INTRODUCTION}

Despite of the large amount of observational data available on radio pulsars, and more recently on X-ray and $\gamma$-ray pulsars, the structure of neutron stars still remains ellusive. Not only the interior is a puzzle, but even the structure of the very surface is a mystery: in the low density - low temperature region near the surface the effects of the magnetic field on the structure of matter and the equation of state still defy any conclusive description. Early work on this question claimed that an iron surface should be a magnetic solid (Ruderman 1974) instead of an atmosphere. Improved calculations in this direction showed that the problem is extremely delicate and the situation is not yet clearly resolved (see, e. g., Neuhauser et al. 1986 and Kössl et al. 1988). However, the surface itself, or the atmosphere, is not necessarily made of iron but probably consists of light elements. A few grams per $\mathrm{cm}^{2}$ of hydrogen at the surface is sufficient to give an optical depth of unity and could be present due to accretion from the interstellar medium or produced on site from the original iron layer by the bombarding of highly energetic particles generated in the magnetosphere. Moreover, fallback of matter after the supernova explosion can also cover the surface, and its magnetic field, by a layer of light elements (Chevalier 1989; Muslimov \& Page 1995). If hydrogen is present, the heavier elements will sediment due to the enormous gravitational field: this would reduce the study of the surface to the comparatively simpler case of a magnetized hydrogen atmosphere. However, hydrogen may agregate into molecules (Lai et al. 1992) because of the magnetic field, and convection within the atmosphere at low temperature $\left(T \lesssim 10^{5} \mathrm{~K}\right.$, Zavlin et al. 1995a) may mix the hydrogen with the heavier elements laying beneath it, complicating again the problem.

Thank to deep ROSAT observations, we now have strong evidence that thermal radiation from four nearby neutron stars has been detected (Ögelman 1995): we are at long last seing the surface of a neutron star. Fitting of these observed thermal spectra with theoretical spectra rised the hope of allowing us to select the correct atmosphere or surface model(s) for these neutron stars. Unfortunately, the energy resolution of the ROSAT PSPC detector and unsolved problems in the calibration of this detector at low energy

prevented this hope to materialize since analyses with various spectra have given equivalent results: spectral fits by themselves are not sufficient to clearly discriminate among the various atmosphere models (Page 1995b; Meyer et al. 1994) with the present observational capability. On the other hand, the strong modulation of the observed pulsed profiles can be interpreted as due magnetic field effects inducing large surface temperature differences (Page 1995a, 1995b, Page \& Sarmiento 1995) and/or anisotropy of the atmospheric emission (Shibanov et al. 1994; Zavlin et al. 1995b). These works thus showed that the shape of the light curves and their energy dependence contain crucial information. 
Presently, the most intriguing and unexplained observed feature is found in Geminga (Halpern \& Ruderman 1993): the amplitude of the pulsations (or pulsed fraction, $P f$ ) in the PSPC channels 8 to 28 (i.e., roughly at energies below $300 \mathrm{eV}$ ) is much larger than in channels 28 to 53 : $33 \%$ vs. 20\%. Blackbody emission always gives an increase of $P f$ with energy (Page 1995a, Page \& Sarmiento 1995). This 'Geminga effect' thus requires the inclusion of magnetic effect on the emitted spectrum and not only on the surface temperature distribution. The preliminary results of Shibanov et al. (1994) showed that realistic atmospheric models are able to produce a slight decrease of $P f$ with increasing energy, but still much smaller than what is observed.

We present here a simple model which is able to produce this decrease in $P f$ in a very natural way by assuming that the surface of Geminga consists of regions with very distinct physical properties (§2). Our results are presented in $\S 3$. We discuss in $\S 4$ some possible reasons for such a surface structure and conclude in $\S 5$.

\section{THE MODEL}

We model the surface of the Geminga pulsar as made of two uniformly magnetized plates, containing the 'north' and 'south' magnetic poles, surrounded by a nonmagnetized crust. This can be considered either as a quasi realistic structure within the scenario of plate tectonics (see $\S 4$ ) or as a schematic representation of a continuous but very nonuniform distribution of surface magnetic field. It has the advantage of simplicity, requiring only two models of atmosphere. Considering Geminga as an orthogonal rotator (Halpern \& Ruderman 1993), the two magnetic plates are located near to the rotational equator. If the surface temperature is determined by the heat flow from the hot interior through the crust, the magnetized plates must be warmer than the remainder of the surface (Page 1995a) and the presence of a single peak in the observed soft X-ray light curves imply that the plates are close to each other. We assume that the whole stellar surface is covered by a hydrogen atmosphere, magnetized on the plates. The plates and the off-plate region have thus very distinct emission properties: the off-plate region has a much harder spectrum, i.e., a large excess in the Wien tail, compared to the plate spectrum due to the different frequency dependence of the opacity (Shibanov et al. 1992). We use the emission spectra calculated by Pavlov et al. (1994) which are similar to the spectra used to perform spectral fit for Geminga by Meyer et al. (1994).

The parameters of our model are thus the followings:

- Diameter and position of the two magnetic plates. 
- Temperature of the off-plate region $T_{o p}$ and magnetic field $B_{p}$ and temperature $T_{p}$ of the plates.

- Mass $M$ and radius $R$ of the star.

- Distance $D$ of the star, interstellar column density $N_{H}$ and orientation of the observer with respect to the star's rotation axis $\zeta$.

We fix $M$ at $1.4 M_{\odot}$ and $R$ at $10 \mathrm{~km}$ and take $\zeta=90^{\circ}$. The temperature $T_{o p}$ (and to some extent $T_{p}$ ) as well as $D$ and $N_{H}$ are determined by fitting the spectrum while the size and location of the plates and $B_{p}$ and $T_{p}$ are determined mainly by fitting the shape of the light curves.

\section{RESULTS}

The values of the parameters are found in Fig. 1 which shows the resulting spectrum: the dominance of the plate emission at low energy is clearly seen. Since the plate emission is the cause of the pulsations this naturally implies that the pulsed fraction is strongly decreasing with increasing photon energy as can be seen in Fig. 2. This result depends critically on the presence of (at least) two different emitting regions with a crossover in their spectra. This crossover is due here to the effect of the magnetic field on the plate spectrum which is absent off-plate but other mechanims providing the same spectral properties would give the same results. The most interesting point is that the warm region (the plates) must have a softer spectrum than the cold one. This is the contrary of what one would expect if the whole surface of the star were homogenously magnetized or non magnetized: the cold atmosphere would show more intense absorption and have a softer spectrum than the warm region. Our result requires thus a drastic difference in the physical properties of the warm region compared to the cold one, here the presence or absence of the magnetic field.

We show finally in Fig. 3 our fit to the light curves in three channel ranges: the decrease of the amplitude of the pulsations with increasing channel number is clearly seen again in bands 7-28 vs. 28-53. The higher channel band 53-256 (not shown in the figure) corresponds to the hard tail which has a different origin than the thermal emission from the whole surface (Halpern \& Ruderman 1993) and we do not attempt to model it. It was proposed by Halpern \& Ruderman (1993) that the decrease in the pulsed fraction of the surface thermal component may be caused by contamination from this hard tail, whose pulses are $105^{\circ}$ off-phase with respect to the thermal component. However, the contribution of the hard tail in the channel range 28-53 is one to two orders of magnitude below the contribution from the thermal component, depending if the hard tail is modeled as a power 
law or blackbody spectrum (see Fig. 3 and 6 in Halpern \& Ruderman 1993) and cannot possibly be responsible for the decrease in the pulsed fraction (Page \& Sarmiento 1995).

The above results also give us a clue to the behaviour of the pulsed fraction in other models. The increase in Pf obtained with blackbody spectra (Page 1995a, Page \& Sarmiento 1995) is most certainly due to the fact that the blackbody hardness increases with temperature. The small decrease in $P f$ obtained with magnetized hydrogen spectra and a dipolar surface field (Shibanov et al. 1994) is itself due to the slight softening of these spectra with increasing field strentgh and the fact that the regions with stronger field have a higher temperature.

\section{DISCUSSION}

\subsection{The parameters of the model}

We have obtained similar results using a larger field strength $\left(\sim 10^{13} \mathrm{G}\right)$ or even by using a blackbody spectrum for the plates: both give the same crossover with the non magnetic spectrum at roughly the same energy. The distance $D$ is then larger $(\sim 30 \mathrm{pc})$, with the same $N_{H}$. The distance $D$ and $N_{H}$ we obtain are comparable to the ones obtained by Meyer et al. (1994) to whom we refer for a discussion of this aspect of the problem. The exact values of the parameters moreover depend on the detector's response. We have used the 1992 March 19 response matrix and the 1993 January 12 version implies, e.g., changes of the order of $5 \%$ in temperature and and increase of about $15 \%$ in $N_{H}$. We did not attempt to perform an accurate spectral fit but only tried to show the main possible mechanism able to produce the 'Geminga effect' as observed in the first set (March 1991) of ROSAT observations. Our spectral fit does have an excess in the channel range 50-90: spectra softer than the non magnetized hydrogen one are needed for a more complete study. A detailed fit of both the spectrum and the energy dependent light curves impose very strong constraints on the atmosphere models and models presently available are apparently not sufficient.

The ratio of the onplate to offplate temperatures we need is within the predicted range from models of heat transport in magnetized and non magnetized neutron star envelopes (Van Riper 1988). However at such low surface temperatures the envelope models are extremely uncertain due to the dominating effect of the magnetic field on the equation of state; magnetized atmosphere models are also not very reliable, since the effect of the atomic motion in the magnetic field have not yet been included (Pavlov \& Mészáros 1993). 


\subsection{Reasons for surface inhomogeneity}

The large inhomogenity needed in our model could be attributed to chemical changes at the surface if the surface is solid. If the surface is gaseous or liquid (covered or not by an atmosphere) then meridional flows, induced by the rotation and/or the magnetic field gradient, would very probably homogenize the chemical composition; in this case only magnetic field inhomogeneity could be invoked to produce the needed spectra.

Magnetic field inhomogeneity is thus most probably the major agent in inducing large variation in the emitted spectrum. In our example we invoked regions with and without magnetic field. Other possibilities are the formation of a magnetic solid surface or the presence of magnetic molecules, both depending on the local field strength and temperature (itself depending on the strength of the underlying field). The strong magnetic inhomogeneity may be due, e.g., to the upraising of the interior field by ohmic diffusion (Muslimov \& Page 1995) or to early tectonic activity of the pulsar.

\subsection{Plate tectonics}

The spin-down of the pulsar induces tremendous stress on the crust both by the differential rotation of the crust superfluid neutrons and/or by the outward motion of the core neutron vortices. Release of this stress can occur either by braking of the crust or unpinning of the crust neutron vortices. The theory of plate tectonics (Ruderman 1976, 1991a, b, c) stipulates that fast spinning pulsars can be expected to break their crust before the crust vortices can unpin. Crust breaking and vortex unpinning are natural explanations

for pulsar glitches (Ruderman 1976, 1991c; Alpar 1977) and analyses of the Crab (Link et al. 1992; Alpar et al. 1994) and Vela (Alpar et al. 1993) pulsar glitches indicate that crust braking is occuring in the former while unpinning is sufficient to explain the latter case. These analyses strongly support the general pattern of crust braking in young and/or fast spinning pulsars and vortex unpinning in the slower ones.

As a consequence, neutron stars born with high spin rate can be expected to have undergone a phase of crust braking and thus should keep on their surface the marks of the previous platelets motion (toward the rotational equator) and formation of a new crust between the moving platelets. The mechanism of 'injection' invoked in explaining pulsar statistics (Vivekanand \& Narayan 1981) stipulates that many pulsars are born with large periods: these pulsars may not have gone through a crust breaking phase. Thus the 'marks' 
left by the tectonic activity are not necessarily present in all pulsars. However, if present, these marks should affect the properties of the pulsar surface and its thermal emission, i.e., they should be visible. The model we have presented here finds a natural explanation within this theory of plate tectonic and the 'Geminga effect' may be such a sign of past tectonic activity on Geminga.

\section{CONCLUSIONS}

We have shown that the energy dependence of the amplitude of the observed pulse profile of Geminga's soft X-ray thermal emission can be naturally explained by using a superposition of two very different spectra: a soft component which dominates at energies below $300 \mathrm{eV}$ and a harder component which dominates at higher energies (at energies above $\sim 500 \mathrm{eV}$ the surface thermal emission is hidden behind the hard tail of different origin). The soft component produces the pulsations, i.e., it is emitted by a smaller area than the hard component, and hence the pulsed fraction naturally decreases with increasing photon energy. Since the soft spectrum is emitted by a smaller region than the hard spectrum it very probably implies a higher temperature in this small area to produce the required crossover of these two spectra.

This postulated surface structure may be simply seen as an idealized representation of a smooth but highly nonuniform distribution of the surface magnetic field or as a quasi realistic structure resulting from a past era of tectonic activity on Geminga.

We thank J. H. Halpern for providing us with the ROSAT data of Geminga, as well as G. G. Pavlov, D. G. Yakovlev and A. Sarmiento for discussions. This work was supported by a DGAPA grant No. IN105794 at UNAM. At the Ioffe Institute it was supported by an ESO C\&EE grant No. A-01-068, a RFFR grant No. 93-02-2916 as well as an INTAS grant No. 94-3834. V. E. Z. is grateful to the Max Planck Institut für Extraterrestrische Physik for financial support through the visitor program. Yu. A. Sh. is indebted to UNAM for its hospitality. 


\section{REFERENCES}

Alpar, M. A. 1977, ApJ, 213, 527

Alpar, M. A., Chau, H. F., Cheng, K. S. \& Pines, D. 1993, ApJ, 409, 345

Alpar, M. A., Chau, H. F., Cheng, K. S. \& Pines, D. 1994, ApJ, 427, L29

Chevalier, R. A. 1989 ApJ, 346, 847

Halpern, J. P., \& Ruderman, M. 1993, ApJ, 415, 286

Kössl, D., Wolff, R. G., Müller, E. \& Hillebrandt, W. 1988, A\&A, 205, 347

Lai, D., Salpeter, E. E. \& Shapiro, S. L. 1992, Phys. Rev., A45, 4832

Link, B., Epstein, R. I. \& Baym, G. 1992, ApJ, 390, L21

Meyer, R. D., Pavlov, G. G., \& Mészáros, P. 1994, ApJ, 433, 265

Muslimov, A. \& Page, D. 1995, ApJ, 440, L77

Neuhauser, D., Langanke, K. \& Koonin, S. E. 1986, Phys. Rev. A33, 2084

Ögelman, H. 1995, in Lives of the Neutron Stars, ed. A. Alpar, Ü Kiziloğlu, \& J. van Paradijs (Dordrecht: Kluwer), 101

Page, D. 1995a, ApJ, 442, 273 (e-print \#9407015 at astro-ph@xxx.lanl.gov)

Page, D. 1995b, Sp. Sc. Rev., in press (e-print \#9501033 at astro-ph@xxx.lanl.gov)

Page, D. \& Sarmiento, A. 1995, in preparation

Pavlov, G. G. \& Mészáros, P. 1993, ApJ, 416, 752

Pavlov, G. G., Shibanov, Yu. A., Ventura, J. \& Zavlin, V. E. 1994, A\&A, 289, 837

Ruderman, M. 1974, in IAU Symposium 53 Physics of Dense Matter, ed. C. J. Hansen (Dordrecht: D. Reidel Publishing Company), 117

-, 1976, ApJ, 203, 213

—, 1991a, ApJ, 366, 261

—, 1991b, ApJ, 382, 576

—, 1991c, ApJ, 382, 587

Shibanov, Yu. A., Zavlin, V. E., Pavlov, G. G. \& Ventura, J. 1992, A\&A, 266, 313

Shibanov, Yu. A., Pavlov, G. G., Zavlin, V. E., Qin, L. \& Tsuruta, S.. 1994 Contribution at the XVII Texas Symposium on Relativistic Astrophysics, Garching

Van Riper, K.A. 1988, ApJ, 329, 339 
Vivekanand, M. \& Narayan, R. 1981, J. Astrophys. Ast., 2, 315

Zavlin, V. E., Pavlov, G. G., \& Shibanov, Yu. A. 1995a, in preparation

Zavlin, V. E., Shibanov, Yu. A., Pavlov, G. G., \& Ventura, J. 1995b, A\&A, 297, 441 
Fig. 1.- Total spectrum and partial spectra from the two plates and the offplate region. The plate spectrum is much softer than the surrounding's spectrum and dominates at low energy: this results in a strong decrease in the pulsed fraction with increasing photon energy as shown in figure 2. The circular magnetized plates have diameters of $60^{\circ}$ and $85^{\circ}$ and their centers are located on the rotational equator at a distance of $140^{\circ}$ from each other. The magnetic field strength on both plates is $4.7 \cdot 10^{12} \mathrm{G}$. The temperature of the plates is $T_{p}=4.17 \cdot 10^{5} \mathrm{~K}$ while the main surface is at $T_{o p}=2.18 \cdot 10^{5} \mathrm{~K}$. The star is at a distance of $15 \mathrm{pc}$ and the effective hydrogen column density is $N_{H}=3 \cdot 10^{20} \mathrm{~cm}^{-2}$. (The two absorption edges at 0.284 and $0.532 \mathrm{keV}$ are due to interstellar $\mathrm{C}$ and $\mathrm{O}$ respectively).

Fig. 2.- Pulsed fraction of the model as detected through the ROSAT PSPC and as would be recorded by a perfect spectrometer. We used the 1992 March 19 version of the PSPC's response matrix.

Fig. 3.- Comparison of the theoretical light curves with the observed pulse profiles of Geminga (Halpern \& Ruderman 1993). Line styles are as in Figure 1. Notice the strong decrease in the pulsation amplitude in the channel range $28-53$ compared to $7-28$. 


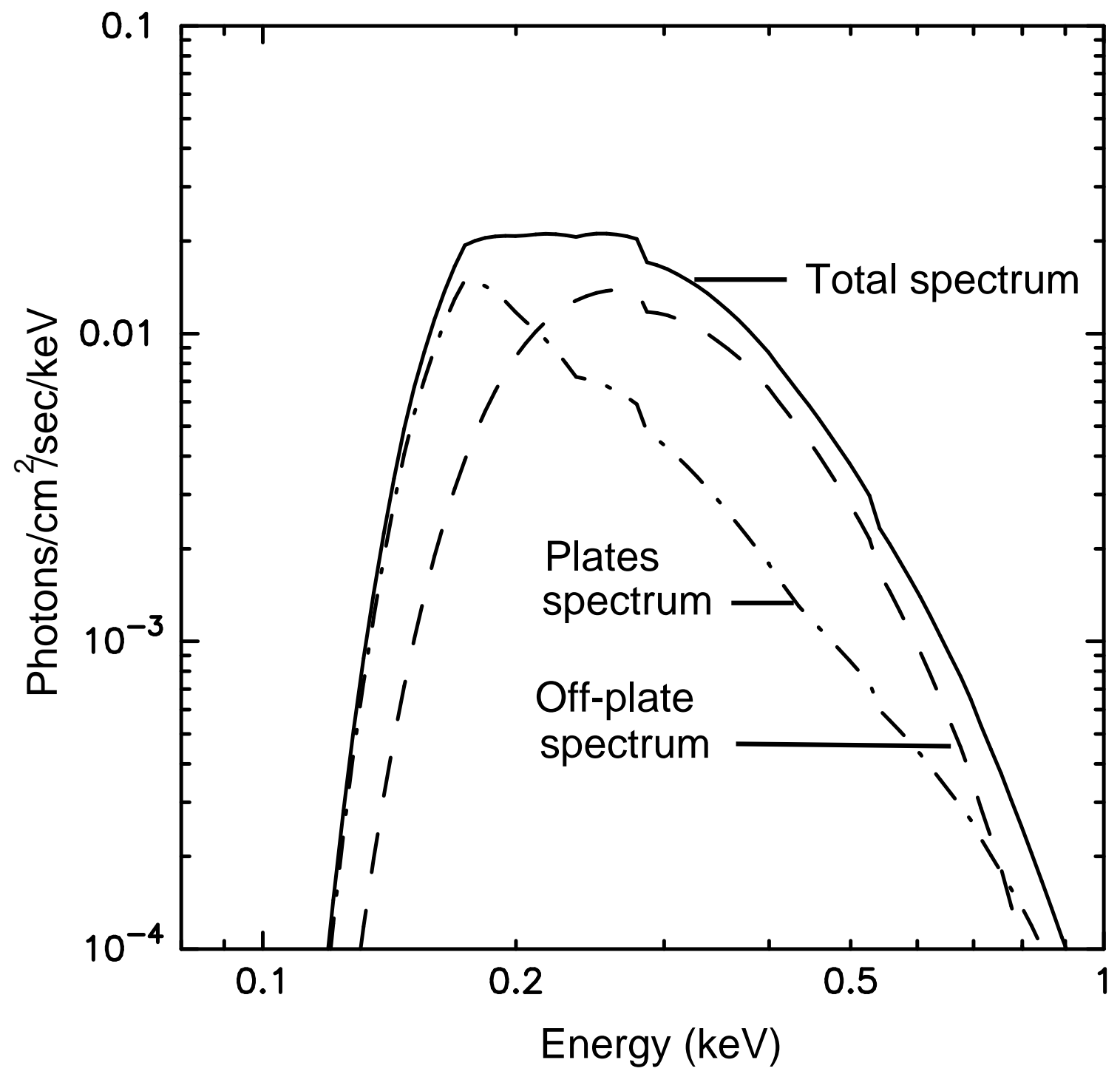

Page, Shibanov \& Zavlin

FIGURE 1 


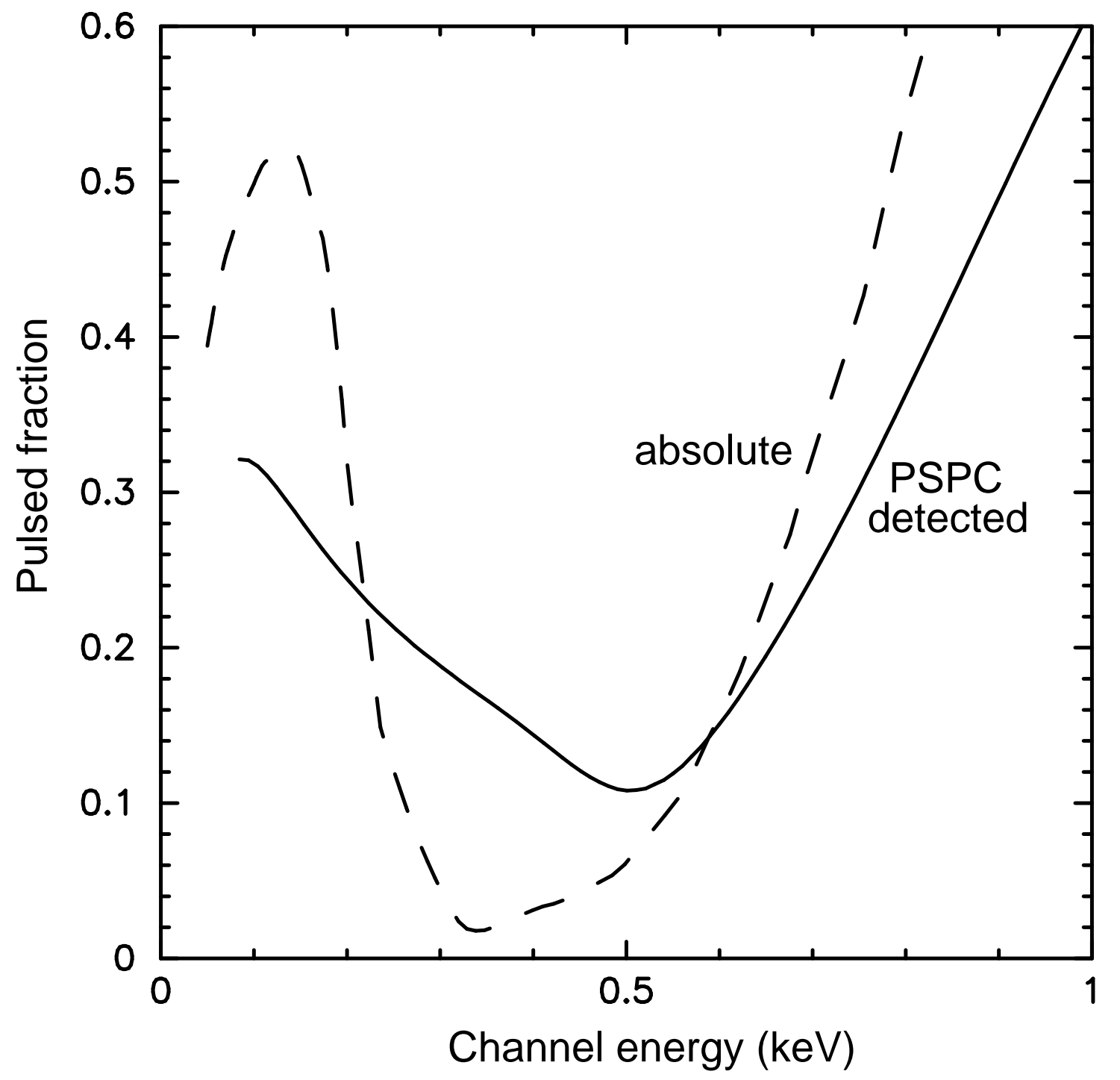

Page, Shibanov \& Zavlin

FIGURE 2 


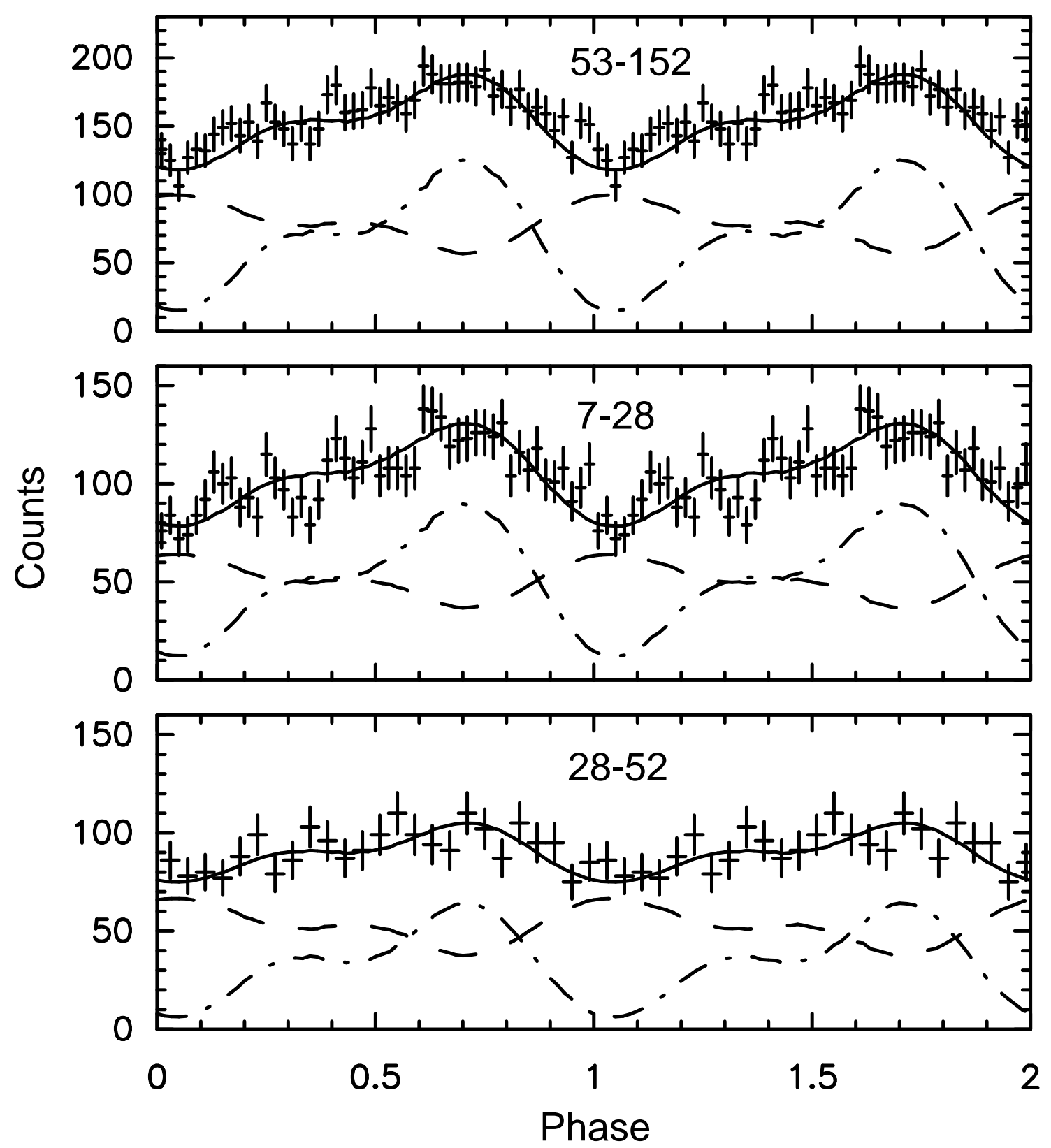

Page, Shibanov \& Zavlin

FIGURE 3 